\title{
A Comparative Study of Epidural, Bupivacaine with Buprenorphine and Bupivacaine with Fentanyl in Lower Limb Surgeries
}

\author{
Dr Santosh Kumar, Dr Lokesh Kumar KS, Dr Rajalakshmi J \\ Dept of Anesthesiology, Dr BR Ambedkar Medical College and Hospital, Bangalore
}

\begin{abstract}
Background

This prospective randomised study was done to compare effects of epidural bupivacaine with buprenorphine over epidural bupivacaine with fentanyl for lower limb surgeries.

Methods

60 patients in the age group 20-60 years belonging to ASA I-II posted for elective lower limb surgeries were divided into two groups of 30 each and studied.Group A received $0.5 \%$ Bupivacaine $15 \mathrm{ml}$ with 150 ug Buprenorphine.Group B received 0.5\% Bupivacaine $15 \mathrm{ml}$ with 50ug Fentanyl. Intraoperatively, sensory and motor blockade, quality and duration of Postoperative analgesia, hemodynamic and respiratory parameters, side effects like nausea, vomiting, respiratory depression, pruritus were studied.

Results

Both groups had faster onset of sensory and motor blockade. Duration of analgesia was significantly longer in Group Athan Group B (766.6 vs 471 mins). The incidence of Nausea and vomiting was more in group A (40 \%) compared to group B (10\%) and pruritus was more in group B (10\%) compared to none in group A.

Conclusion

It is concluded that epidural buprenorphine is better in providing prolonged satisfactory postoperative analgesia as compared to epidural Fentanyl.
\end{abstract}

\section{Introduction}

The International Association for the Study of Pain defines pain as "an unpleasant sensory and emotional experience associated with actual or potential tissue damage, or described in terms of such damage". Other than psychological trauma, pain is shown to affect the physiology of almost all the system including respiratory, cardiovascular and metabolic profile there by increasing the morbidity. ${ }^{1}$

It is possible to perform all surgical procedures while the patient is under GA, but GA has its own disadvantages and limitations; therefore regional anesthesia is preferred for lower extremity surgeries. The advantages of regional anesthesia are anawake patient,polypharmacy avoided, no airway manipulation, good motor and sensory blockade, early food intake by the patient, less incidence of PONV, prolonged postoperative analgesia and ideal operating conditions.

Intrathecalanesthesia and epidural anesthesia (EA) are the most popular regional anesthesia techniques used for surgeries below umbilicus. EA is more versatile in providing anesthesia, analgesia and treatment of chronic disease syndromes. It provides better postoperative pain control and more rapid recovery from surgery. It also provides effective prolonged surgical anesthesia, prolonged post-operative analgesia, reduces the incidence of hemodynamic changes and reduces the incidence of PDPH as the dura is not pierced.

A local anesthetic-opioid combination provides superior analgesia during perioperative period. ${ }^{2}$ This combination limits rapid regression of sensory blockade and possibly decreases the dose of local anesthetic administered. Analgesia provided by epidural opioids is superior to that with systemic opioids. Bupivacaine a local anesthetic iswidely used drug in epidural anesthesia. Buprenorphine is a thebaine derivative, mu-receptor partial agonist and antagonist. It is effective in relieving moderate to severe pain. Fentanyl is a phenylpiperidinederivative synthetic opioid agonist; which is also very effective analgesic.

Hence the present study is designed to compare effectiveness of fentanyl and buprenorphine through epidural route.

\section{Methods}

This is a prospective randomized study to compare between epidural, bupivacaine with buprenorphine and bupivacaine with fentanyl in lower limb surgeries. After Ethical committee clearance and informed consent of the patients, a total of 60 patients of either sex aged between 20-60 years belonging to ASA Grade I \& II scheduled for elective lower limb surgeries were randomly selected. 
The exclusion criteria included pregnant women, Patients with H/o Cardio-Respiratory disorders, Hepatic and Renal diseases, convulsions \& neurological deficits, Spinal deformities, Psychiatric diseases, ASA Grade III \& above and patients with contra-indications for epidural anesthesia.

The grading of postoperative pain is done using VNS (verbal numerical scale).

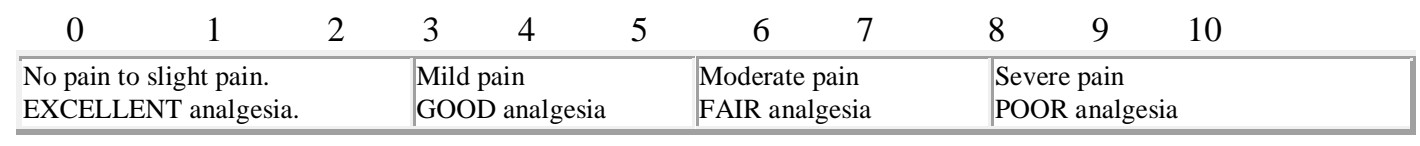

On the day of surgery patients connected to multichannel monitor and baseline heart rate, non-invasive blood pressure and $\mathrm{SpO}_{2}$ was recorded. After insertion of $18 \mathrm{G} \mathrm{IV} \mathrm{cannula,} \mathrm{patients} \mathrm{were} \mathrm{preloaded} \mathrm{with} \mathrm{ringer}$ lactate $500 \mathrm{ml}$ over 30 minutes.

Under strict aseptic precautions, epidural space was identified in L2-3 interspace using 18GTuohy's needle by loss of resistance technique, epidural catheter was inserted $3 \mathrm{~cm}$ inside epidural space and secured in place, continuous monitoring was done.A test dose of $3 \mathrm{ml}$ of $2 \%$ lignocaine with adrenaline $(1: 2,00,000)$ was given to rule out intravascular or intrathecal placement. 5 minutes after test dose, in the absence of any adverse sequelae, $16 \mathrm{ml}$ of study drug was injected depending on patient study group through epidural catheter. After adequate blockade $\left(\mathrm{T}_{10}\right)$ patient was repositioned based on surgical requirements.

Patients were divided into two groups:

Group A Buprenorphine with Bupivacaine group - 0.5\% Bupivacaine $15 \mathrm{ml}$ (75mg) with $0.5 \mathrm{ml}$ (150 ug) Buprenorphine (preservative free) with $0.5 \mathrm{ml}$ sterile normal saline made to a total of $16 \mathrm{ml}$.

Group B Fentanyl With Bupivacaine group - 0.5\% Bupivacaine $15 \mathrm{ml}$ (75mg) with $1 \mathrm{ml}$ (50ug) Fentanyl (preservative free).

Intra operatively assessment of sensory and motor blockade was done at the end of each minute after injecting $16 \mathrm{ml}$ of the study drug. The onset time and the time for maximum motor and sensory block and the maximum level of sensory and motor block were recorded.

Sensory blockade was assessed using a short beveled 22 gauge needle andMotor blockade in the lower limbs was assessed using modified Bromage scale.
0 - no motor blockade
1- Inability to raise extended leg
2 - Inability to flex the knee
3 - Inability to flex ankle joint

Vital parameters such as the HR, BP, RR and oxygen saturation were continuously monitored for every $5 \mathrm{~min}$ for first $15 \mathrm{~min}$ and every $15 \mathrm{~min}$ throughout intraoperative period and every half an hour in the postoperative period for 2 hours.

Postoperatively observations were made regardingduration of post-operative analgesia, quality of postoperative analgesia (VNS), hemodynamic monitoring (NIBP \& HR), the need for rescue analgesic supplementation, episodes of intraoperative and postoperative side effects such as hypotension, bradycardia, desaturation $\left(\mathrm{SpO}_{2}<90 \%\right)$ and respiratory depression ( $<10$ breaths per minute), pruritus, nausea vomiting noted and treated.

\section{Results}

A total of 60 patients of either sex randomly selected for the study. Statistical data was analysed using Chi-square test, Student t-test (Paired and unpaired t-test).

\section{Demographic Data Analysis}

Groups were comparable regarding age, gender, weight and height.

\section{Onset Of Analgesia}

It was observed that onset of analgesia in Group-A was 7.56 min when compared to Group-Bwhich was $6.6 \mathrm{~min}$, which is statistically insignificant $(\mathrm{P}<0.05)$.

The mean time to achieve complete motor blockade was 18.9 min in group A and 18.63 in group B which was statistically insignificant in both the groups. 


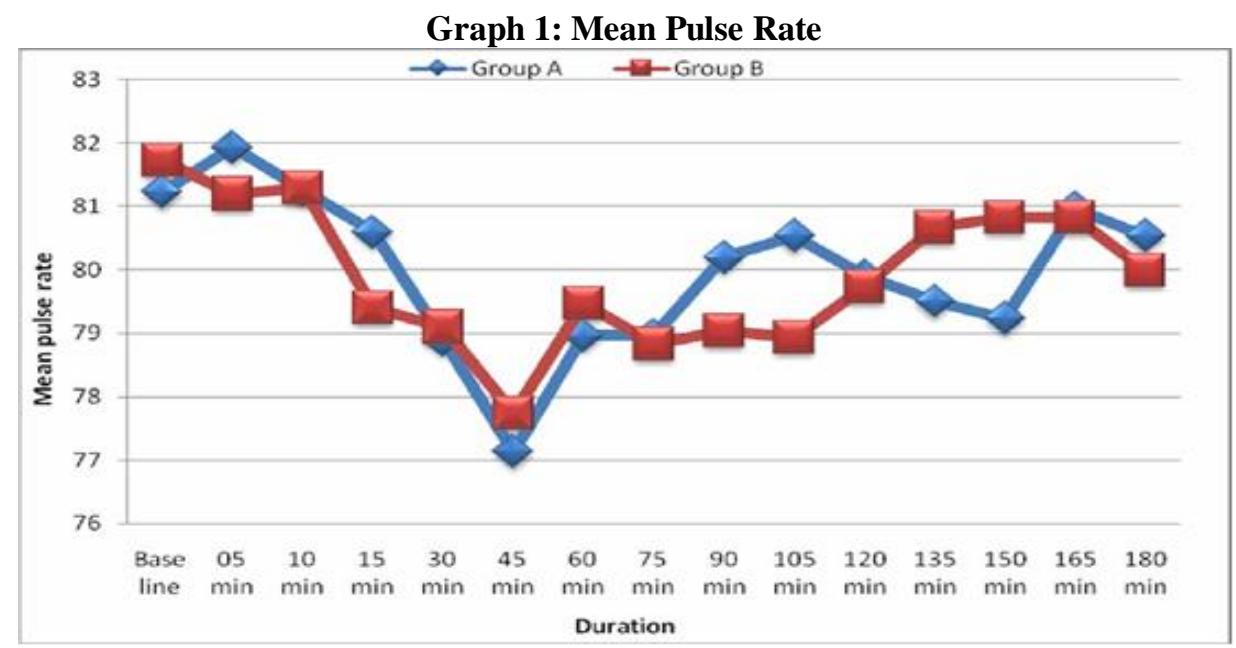

There was moderate drop in the pulse rate in $30 \mathrm{~min}$ and $45 \mathrm{~min}$ in both the groups which was statistically insignificant.

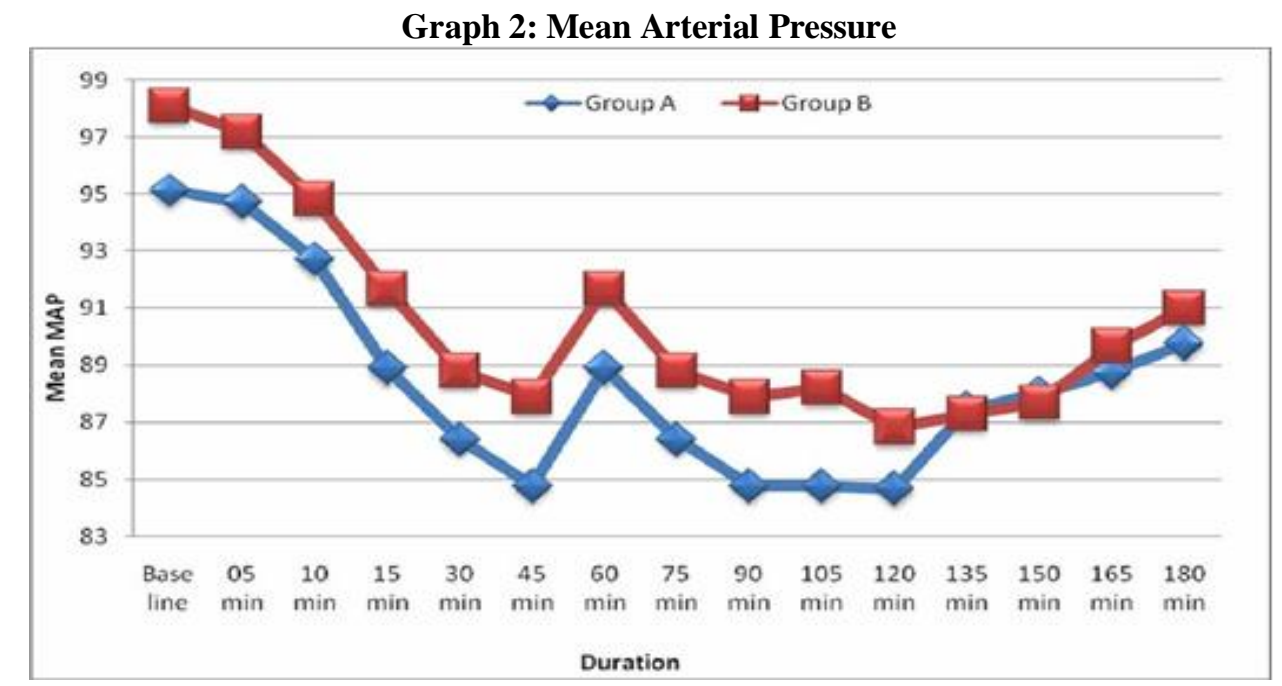

FChange $=35.694 \mathrm{P}>0.05$ insignificant

FChange $\mathrm{x}$ groups $=0.993 \mathrm{P}>0.05$ insignificant

It can be seen from the graph no. 2 that change in MAP was not significant at any time interval in between the two groups.

Graph 3: Variation In Respiratory Rate Per Minute Within Each Group And Inbetween The Groups

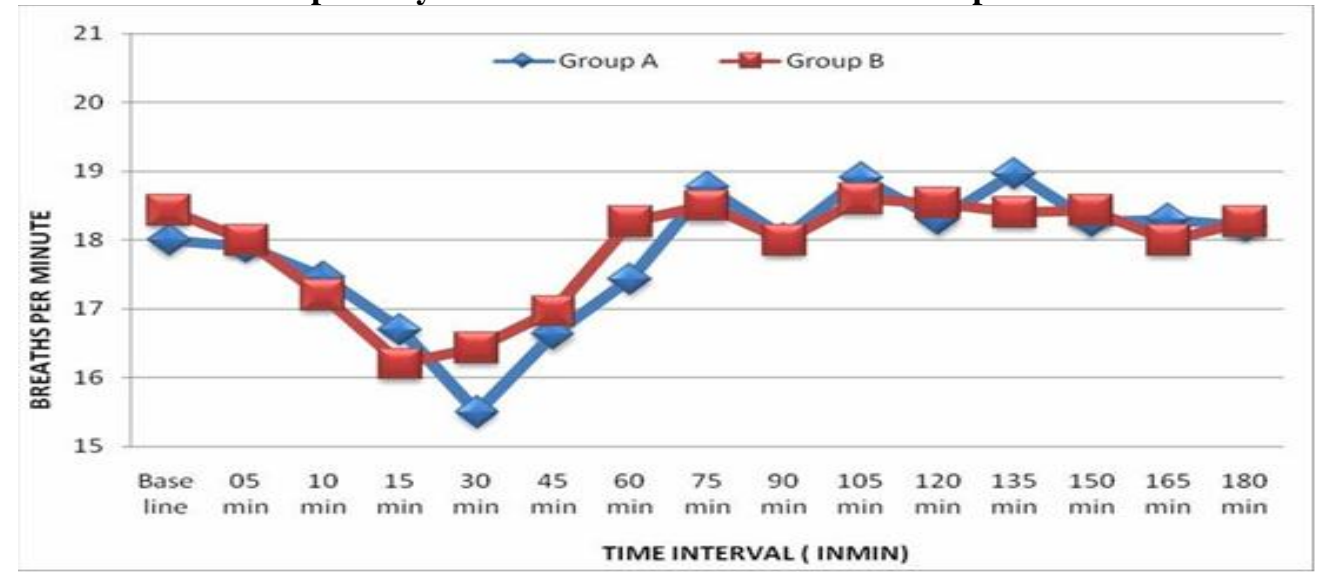


There was insignificant drop in respiratory rate in both the groups at 10, 15, $30 \mathrm{~min}$. This was due to the respiratory depressant action of both the drugs.

Table 1: Mean Duration Of Analgesia

\begin{tabular}{|c|c|c|c|c|c|}
\hline & $\begin{array}{c}\text { NO OF } \\
\text { PATIENTS }\end{array}$ & $\begin{array}{c}\text { MEAN } \\
\text { DURATION } \\
\text { (inmin) }\end{array}$ & SD & $\mathbf{t}$ & SIGNIFICANCE \\
\hline GROUP A & 30 & 766.6 & 169.67 & \multirow{2}{*}{7.178} & P $<0.05 \mathrm{~S}$ \\
\hline GROUP B & 30 & 471 & 148.68 & \\
\hline
\end{tabular}

SD: Standard Deviation, S: Significant

Duration of analgesia was time at which, patients complained of pain of 5 and above on the verbal numerical scale was noted. That point was taken as the end of fair analgesia and at that point, top up doses were given based on requirement. It can be noted from the above table that duration of analgesia was significantly longer in Group A with mean duration of 766.6 minutes as compared to $471 \mathrm{~min}$ in Group B. $(\mathrm{p}<0.05)$

\section{Side Effects}

It was noted that incidence of nausea was $30 \%$ in group A compared to $10 \%$ in group B. Incidence of vomiting was $10 \%$ and pruritus $0 \%$ in group A when compared to $0 \%$ and $33.3 \%$ respectively in group B.

\section{Discussion}

Pain is a more terrible lord of mankind than death itself. Satisfactory pain relief has always been a difficult problem in clinical practice. It is found that operative pain is more severe after surgery and thereafter gradually diminishes over the next 24 hours. Epidural anesthesia is superior to Spinal and hence is widely being used especially in patients undergoing surgical procedures involving lower parts of the body. To fulfil this demand, there is a need for local anaesthetic with desirable properties like longer duration of sensory blockade and shorter duration of motor blockade. ${ }^{4}$

Traditionally epidural bupivacaine $(0.125 \%)$ is used for post-operative analgesia.The discovery of opioid receptors in the spinal cord made it clear that epidural administration of opioids is superior to traditional intravenous and intramuscular injections of opioids.

Hence a study entitled, a comparative study between epiduralbupivacaine with buprenorphine and bupivacaine with fentanyl in lower limb surgerieswas undertaken to evaluate sensory and motor blocking properties, quality and duration of analgesia and side effects if any.

\section{Onset of Sensory Blockade}

Onset of sensory blockade is taken as the time from the completion of the injection of the study drug till the patient does not feel the pin prick at L1 level on the dependent side.Mean time of analgesia in our study was; Group-A $7.53 \mathrm{~min}$ and Group-B $6.60 \mathrm{~min}$.

Zenz M, Pipenbrocks S, did a double blind comparison of epidural Buprenorphine $(0.15 \mathrm{mg})$ and epidural morphine $(4 \mathrm{mg})$ for postoperative pain relief. Buprenorphine produced analgesia with short latency 6.8 min. This is close to our observation of $7.53 \mathrm{~min} .{ }^{3}$ High lipid solubility and high potency may explain the faster onset of pain relief in buprenorphine group.

SurajDhale and VaishaliShelgaonkar in 2000, studied different doses of epidural fentanyl $(25 \mu \mathrm{g}, 50 \mu \mathrm{g}$, $75 \mu \mathrm{g}$ ) with $0.5 \%$ bupivacaine for perioperative analgesia found that $50 \mu \mathrm{g}$ had a quicker onset of analgesia within 9.53 min which is close to our observation. ${ }^{4}$

\section{Duration of Analgesia}

Duration of analgesia is taken from the time of injection till the patient complains of pain at the site of surgery. Time at which, patients complained of pain more than 5 and above on the verbal numerical scale was noted.

In our study mean duration of analgesia in group A was 766 min which was significantly longer compared to group B which was $471 \mathrm{~min}$.

In their comparative study between epidural buprenorphine and epidural Ketamine for postoperative pain relief D Kumar, N Dev and N Gupta found that $0.15 \mathrm{mg}$ Buprenorphine with $10 \mathrm{ml}$ of $0.9 \%$ saline had 
longer duration of action 13.1 hours (range 8-12 hours) compared to $10 \mathrm{mg}$ of Ketamine with $10 \mathrm{ml}$ of $0.9 \%$ saline, which had mean duration of 5.2 hours. ${ }^{5}$ In our study mean duration of analgesia in Group A was 766 min (12 hours).

Longer duration of action and analgesia efficacy of epidural buprenorphine can be explained by its high affinity for spinal receptors. Higher lipid solubility of buprenorphine favors its diffusion in to spinal cord. The diffusion from the spinal cord in to the blood stream is slow and does not approach the bulbar Centers. Hence high lipid solubility, strong opiate receptor binding and intense and prolonged activity was responsible for longer duration of action. ${ }^{6}$

Higher lipophilicity of fentanyl results in shorter duration of action, lower incidence of side effects, and reduced risk of respiratory depression.?

\section{Motor Blockade}

Onset of motor blockade is taken from the completion of the injection of study drug till the patient develops modified Bromage scale grade 1.

Time for maximum motor blockade is defined as the time from the completion of the injection of the study drug to the maximum motor blockade attained.

The mean time to achieve complete motor blockade was $18.9 \mathrm{~min}$ in group A and 18.63 which was statically insignificant in both the groups.

SurajDhale and VaishaliShelgaonkar, in 2000 studied different doses of epidural fentanyl $(25 \mu \mathrm{g}, 50 \mu \mathrm{g}$, $75 \mu \mathrm{g}$ ) with $0.5 \%$ bupivacaine for perioperative analgesia where mean onset of motor blockade was $26.13 \pm 1.80$ $\min ^{4}$

\section{Hemodynamic Changes}

The mean HR reduction indicating analgesia was also insignificant in both the groups. The mean base line heart rate in group A which was $81.233 /$ min reduced gradually to 78.966 at $1 \mathrm{hr}$ and remained stable throughout the study. The mean baseline heart rate thatwas $81.733 / \mathrm{min}$ group B reduced to $78.8 / \mathrm{min}$ at $60 \mathrm{~min}$ then significantly remained unchanged throughout the study.

The reduction in MAP was statistically insignificant in both groups. In group A, MAP from base line $95.13 \mathrm{mmHg}$ fell to $84.8 \mathrm{mmHg}$ at $45 \mathrm{~min}$. Then picked up to $88 \mathrm{mmHg}$ at $150 \mathrm{~min}$ remained same throughout the study. In group B, MAP from baseline $98.97 \mathrm{mmHg}$ fell to $87.90 \mathrm{mmHg}$ at $45 \mathrm{~min}$ then picking up slowly to $93.7 \mathrm{mmHg}$ at $120 \mathrm{~min}$ thereafter remained significantly high throughout the study but the difference was not significant in both the groups $(\mathrm{P}>0.005)$.

UshaRathi, M. Singh, M.Pramanik in 1993 studied postoperative analgesic efficacy with different doses extradural buprenorphine for herniorrhaphy and found that buprenorphine in $0.3 \mathrm{mg}$ dose is suitable for single shot epidural injection which provides both anaesthesia and postoperative analgesia with hemodynamic stability which correlates with our observation. ${ }^{8}$

\section{On Respiratory Rate}

In our study mean base line respiratory rate in Group A fell from $18 / \mathrm{min}$ to around 15.5 in 30min gradually picking up by $90 \mathrm{~min}$ and remained to $18.2 / \mathrm{min}$. InGroup B, mean basal respiratory rate which was $18.4 / \mathrm{min}$ fell to 16.43 at $30^{\text {th }} \mathrm{min}$, picked up to $18 / \mathrm{min}$ at $90 \mathrm{~min}$ which is again comparable without any significant difference.There was no severe respiratory depression in either group.This is in concurrence with studies done by Zenzet $\mathrm{al}^{3}$ and Lytle SA et $\mathrm{al}^{9}$.

\section{Side Effects}

The four classic side effects of neuraxial opioids are Pruritus, Nausea and vomiting, Urinary retention and Depression of ventilation. Side effects are caused by the presenceof drug either in CSF or systemic circulation.

In our study 9 patients (30\%) developed nausea and 2 patients (10\%) had vomiting in group A and in group B only 2 patients $(10 \%)$ developed nausea with no vomiting.Observations of study done by D. Kumar ${ }^{5}$ and co-workers and Hayashi $\mathrm{H}$ and co-workers ${ }^{10}$ correlate with our study on Buprenorphine.Opioids produce nausea and vomiting by direct stimulation of CTZ in the area postrema of the medulla.

In our study Group B, 10 patients (33.3\%) developed pruritus compared to none in Group A. Studies done by SA Lytle et al. correlates with our study on fentanyl. ${ }^{9}$ Pruritus may be generalized but is more likely to be localized to the face, neck, or upper thorax. It is likely due to cephalad migration of the opioids in CSF and subsequent interaction with opioid receptors in trigeminal nucleus.

\section{Conclusion}

From our study it can be concluded that epidural buprenorphine is better in providing prolonged 
satisfactory postoperative analgesia as compared to Fentanyl.Regarding the side effects, the incidence of nausea and vomiting was more in buprenorphine as compared to fentanyl group.

\section{Bibliography}

[1]. Dr.Nirvik Pal, Dr.Kiran Malhotra, Dr.Chitra et al. Effect of morphine on postoperative respiratory functions: Comparision between systemic and epidural routes. Indian J. Anaesth 2004;48(3):204-207

[2]. RG Wheatley, SA Schug, D. Watson. Combination of local anaesthetic and an opioid combination. Br J Anaesth 2001;87:47

[3]. Zenz M., Pipen brock S., Hubner B., Glocke M: 'A double blind comparison of epidural Buprenorphine and epidural morphine in post-operative pain'. Anaesth - Intensive therapy - Notfallmed, 16(6): 333-9, 1981

[4]. SurajDhale, VaishaliShelgaonkar and VV Akulwar. A comparative study of epidural bupivacaine and epidural bupivacaine with fentanyl for peri operative analgesia. Indian journal anaesthesia 2000;44:35

[5]. D Kumar,Dev Gupta. Comparative study of Epidural Buprenorphine and Ketamine for postoperative pain relief. Ind. Journal Anaesthesia1997; 41:38-49.

[6]. Cahill J, Murphy D, O'Brien D, Mulhall J. Epidural buprenorphine for pain relief after major abdominal surgery. A controlled comparison with epidural morphine. Anaesthesia. 1983 Aug;38(8):760-4

[7]. JW Downing. BUPRENORPHINE: A new potent long-acting synthetic analgesic. Comparison with morphine.Br. J. Anaesth. (1977) 49 (3):251-255.

[8]. UshaRathi, M.Singh.Evaluation of different doses of Buprenorphine given epidurally with Lignocaine to relieve postoperative pain $1993 ; 41: 122-124$.

[9]. SA Lytle, DM Goldsmith, TL Neuendorf, and ME Lowry Postoperative analgesia with epidural fentanyl. Journal of the American Osteopathic Association, Vol 91, Issue 6, 547-547 (1991)

[10]. Hayashi H, Nishiuchi T, Tamura H, Takeda K. Comparison of buprenorphine and fentanyl for postoperative pain relief by continuous epidural infusion. Masui. 1993 Dec;42(12):1763-8 